\title{
Does Patient Demand Affect the Physicians' Decision to Prescribe Antibiotics in Emergency Departments? A Survey Study
}

\author{
Gulsah Cikrikci Isik (D), Evren Ekingen (D) \\ Clinic of Emergency, Batman Region Public Hospital, Batman, Turkey
}

Cite this article as: Cikrikci Isik G, Ekingen E. Does Patient Demand Affect the Physicians' Decision to Prescribe Antibiotics in Emergency Departments? A Survey Study. Eurasian J Emerg Med. 2018; 17 (4): 171-7.

\begin{abstract}
Aim: Antibiotic resistance is a growing public health problem, and one of the important reasons for this is an inappropriate prescription of antibiotics. The aim of this study was to evaluate the knowledge and perceptions related to antibiotic prescription among physicians in the emergency department (ED) and also to find out if the patient demand affects the physicians' decision to prescribe antibiotics.
\end{abstract}

Materials and Methods: A cross-sectional survey of physicians working at ED. The study was conducted during 2017 with an online questionnaire, and results were analyzed by Statistical Package for Social Sciences (SPSS).

Results: Out of 282 relevant questionnaires, in $62.1 \%$ of them, the participants think that inappropriate antibiotic prescription was frequent, and some even mentioned that they sometimes (39\%) prescribed antibiotics inappropriately. The awareness of antibiotic stewardship was poor in half of the participants. More than two-thirds of the physicians noted that patients had been forcing them to prescribe antibiotics; and physicians who were newer at the profession ( $<5$ years) noted that this was affecting their decision more often (31.5\%). Among other factors, need for making quick decisions at ED and overcrowding of the ED leading to inappropriate antibiotic prescription, and there was no relationship between the responses and professional seniority $(p=0.7$ and $p=0.1$, respectively), but there was an inverse relation between clinical practice and overcrowding $(p=0.01)$.

Conclusion: Our study demonstrated that all physicians thought antibiotics had been prescribed inappropriately. Patient demand, need for making quick decisions, and overcrowding of EDs are some of the factors that affect the antibiotic prescription decisions of physicians, and the effect of these factors was inversely proportional to increased clinical practice.

Keywords: Antibiotic, inappropriate, demand, overcrowding

\section{Introduction}

Although the history of antibiotics is not very old, antibiotic resistance has already become a major problem that threatens human health worldwide. According to data of the World Health Organization, if no preventive measures are taken, by 2050, 10 million deaths may be attributable to antimicrobial resistance all around the world (1).
Despite previous reports showing the "slowly emerging disaster" related to antibiotic resistance, there is evidence of continuing overprescription (2). A 2013 report by the Organization for Economic Cooperation and Development has identified the countries with the highest antibiotic consumption rate: Turkey is in the first place with a defined daily dose of 42.2 per 1,000 people, followed by Greece, France, and Italy (3).

ORCID IDs of the authors: G.C.I. 0000-0002-6067-7051; E.E. 0000-0003-4895-2345. 
Antibiotic overuse, incorrect dosing, and extended duration are some of the leading causes of antibiotic resistance (4). Inappropriate antibiotic prescription is a public health problem and is most commonly seen among patients with upper respiratory tract infections (URTI) (5). It is known that physicians are influenced by many factors when prescribing a certain drug unnecessarily, among which the chief factor is the aim of pleasing the patient (2). Accordingly, patient demand is a significant factor for a physician's drug prescription (5). To tackle the current worldwide bacterial resistance crisis, antibiotic stewardship programs, which are a set of interventions aiming at prescribing antibiotics appropriately and responsibly, have come to the fore (6).

The aim of this study was to examine the antibiotic prescription habits of physicians (general practitioners (GP), emergency medicine (EM) residents; and emergency medicine specialists) working in emergency departments (EDs) and also to investigate their awareness related to antibiotic resistance and knowledge levels related to antibiotic stewardship programs. Furthermore, we aimed

Table 1. Characteristics of participants and indications, and group of antibiotics commonly prescribed

\begin{tabular}{|c|c|}
\hline Variable & Number (\%) \\
\hline \multicolumn{2}{|l|}{ Gender } \\
\hline Male & $156(55.3)$ \\
\hline Female & $126(44.7)$ \\
\hline \multicolumn{2}{|l|}{ Professional Status } \\
\hline General practitioner & $92(32.6)$ \\
\hline Emergency medicine resident & $50(17.7)$ \\
\hline Emergency medicine specialist & $140(49.6)$ \\
\hline \multicolumn{2}{|l|}{ Duration of clinical practice (years) } \\
\hline $0-5$ & $73(25.9)$ \\
\hline $5-10$ & $113(40.1)$ \\
\hline $10-15$ & $59(20.9)$ \\
\hline $15-20$ & $26(9.2)$ \\
\hline $20+$ & $11(3.9)$ \\
\hline \multicolumn{2}{|c|}{ Indications antibiotics most commonly prescribed for } \\
\hline $\mathrm{URTI}^{*}$ & $171(60.6)$ \\
\hline LRTI* & $64(22.7)$ \\
\hline $\mathrm{UTI}^{*}$ & $41(14.5)$ \\
\hline $\mathrm{AGE}^{*}$ & $3(1.1)$ \\
\hline Others & $3(1.1)$ \\
\hline \multicolumn{2}{|c|}{ Group of antibiotics most commonly prescribed } \\
\hline Penicillin & $160(56.7)$ \\
\hline Cephalosporin & $90(31.9)$ \\
\hline Macrolide & $17(6)$ \\
\hline Quinolone & $13(4.6)$ \\
\hline Others & $2(0.7)$ \\
\hline
\end{tabular}

URTI: upper respiratory tract infection; LRTI: lower respiratory tract infection; UTI: urinary tract infection; AGE: acute gastroenteritis to investigate whether increased patient demand for antibiotics was affecting the physician's opinion during prescription and to consequently determine if there is a relationship between this situation and the clinical practice of the physician.

\section{Materials and Methods}

This was a cross-sectional survey study, and it was approved by and carried out in accordance with the regulations of the local research ethics committee. Physicians at different levels of professional seniorities, who were GPs, EM residents, and EM specialists, working in EDs of hospitals were invited to participate. There was no informed consent form because the invitation was sent either by e-mail, SMS messages, or via social media groups. Further, no personal identifying information was required; all email addresses and phone numbers were kept secret and strict confidentiality was maintained; and participation was voluntary. An online questionnaire, including 22 items, was created in an easily accessible, smartphone-friendly website. Demographic data including gender, age, professional status (GP, EM resident, or EM specialist), and duration of clinical practice were collated. Next, three questions were asked to understand the antibiotic prescription habits of doctors: "How many patients do you see in a day?"; "For which indication do you prescribe antibiotics most?"; and "Which group of antibiotics do you prescribe most?" Other items focused on the physicians' opinions regarding antibiotic resistance and were as follows: their awareness of antibiotic resistance prevalence, their knowledge of antibiotic stewardship programs, and their beliefs about inappropriate antibiotic prescription. To assess the factors of inappropriate prescription, we asked whether the patients' demands and persistent attitudes, as well as overcrowding and need for making quick decisions at EDs, were affecting the prescription decision of the doctor. The questions about beliefs and attitudes used a 5-point scale for the response options from "always" to "never" and from "excellent" to "poor." Full survey wording is presented in Appendix 1

\section{Statistical analysis}

Statistical analysis was performed using the Statistical Package for Social Science version 15.0 (SPSS Inc.; Chicago, IL, USA). Reliability and validity of the questionnaire were measured by Cronbach's alfa, and it was found to be 0.71 . Demographic data related to participants were expressed as numbers and percentages. Descriptive variables such as age and gender, were categorized, and all the categorical variables were analyzed using the Pearson Chi-square and Fischer's exact test. $p$ less than 0.05 was considered statistically significant.

\section{Results}

Among 299 returned questionnaires, we had 282 completed and relevant ones. More than half of the participants were males $(55.3 \%)$. The sample included 92 (32.6\%) GPs, 50 (17.7\%) EM residents; and $140(49.6 \%)$ EM specialists. Only one-quarter of them $(25.9 \%)$ were relatively new with a clinical practice of fewer than five years (Table 1).

Of all the physicians, $61.3 \%$ see more than 100 patients per day; it was even more than 200 for $31.9 \%$ of them. The most common indication for the prescription of antibiotics was upper respiratory tract infections (URTI) (60.6\%), followed by lower respiratory infections 
Table 2. Relation between the responses and professional status of the physicians

Questions

Professional status

$\mathbf{n}^{*}(\%)$

Do you think that you prescribe antibiotics in accordance with the guidelines for the correct indications?

\begin{tabular}{|l|c|c|c|c|c|c|}
\hline & Never & Rarely & Sometimes & Often & Always & p \\
\hline GP* $^{*}$ & $4(4.3)$ & $4(4.3)$ & $20(21.7)$ & $54(58.7)$ & $14(15.2)$ & $3(6)$ \\
\hline EM* resident & $4(8)$ & $4(8)$ & $9(18)$ & $34(68)$ & 0.324 \\
\hline EM specialist & $15(10.7)$ & $15(10.7)$ & $32(22.9)$ & $74(52.9)$ & $19(13.6)$ & \\
\hline
\end{tabular}

Are you aware of antibiotic stewardship?

\begin{tabular}{|l|c|c|c|c|c|c|}
\hline & Excellent & Good & Average & Below average & Poor & $12(13)$ \\
\hline GP & $13(14.1)$ & $13(14.1)$ & $12(13)$ & $55(59.8)$ & $57(41)$ \\
\hline EM resident & $6(12)$ & $6(12)$ & $9(18)$ & $5(10)$ & 0.034 \\
\hline EM specialist & $19(13.7)$ & $19(13.7)$ & $42(30.2)$ & $21(15.1)$ & $30(60)$ & \\
\hline
\end{tabular}

Does the patient demand affect your antibiotic prescription decision?

\begin{tabular}{|l|c|c|c|c|c|c|}
\hline & Never & Rarely & Sometimes & Often & Always & \\
\hline GP & $13(14.1)$ & $29(31.5)$ & $34(37)$ & $14(15.2)$ & $2(2.2)$ & $3(6)$ \\
\hline EM resident & $7(14)$ & $13(26)$ & $15(30)$ & $12(24)$ & 0.491 & $6(4.3)$ \\
\hline EM specialist & $13(9.3)$ & $53(37.9)$ & $39(27.9)$ & $29(20.7)$ & \\
\hline
\end{tabular}

Do you prescribe antibiotics to avoid discussion with the patient?

\begin{tabular}{|l|c|c|c|c|c|c|}
\hline & Never & Rarely & Sometimes & Often & Always & $7(7.6)$ \\
\hline GP & $15(16.3)$ & $23(25)$ & $22(23.9)$ & $25(27.2)$ & $8(16)$ & $9(18)$ \\
\hline EM resident & $6(12)$ & $8(16)$ & $19(38)$ & 0.011 & $7(5)$ & \\
\hline EM specialist & $12(8.6)$ & $49(35)$ & $39(27.9)$ & $33(23.6)$ & \\
\hline
\end{tabular}

Does need for making quick decisions at the emergency department affect your antibiotic prescription?

\begin{tabular}{|l|c|c|c|c|c|c|}
\hline & Never & Rarely & Sometimes & Often & Always & $6(6.5)$ \\
\hline GP & $7(7.6)$ & $22(23.9)$ & $33(35.9)$ & $24(26.1)$ & $17(34)$ & $5(10)$ \\
\hline EM resident & $4(8)$ & $10(20)$ & $14(28)$ & 0.758 & $8(5.7)$ & \\
\hline EM specialist & $13(9.3)$ & $22(15.7)$ & $52(37.1)$ & $45(32.1)$ & \\
\hline
\end{tabular}

Does the overcrowding at $\mathrm{ED}^{*}$ cause you to prescribe unnecessary antibiotics?

\begin{tabular}{|l|c|c|c|c|c|c|}
\hline & Never & Rarely & Sometimes & Often & Always & $6(6.5)$ \\
\hline GP & $8(8.7)$ & $27(29.3)$ & $25(27.2)$ & $26(28.3)$ & $9(18)$ & $8(16)$ \\
\hline EM resident & $2(4)$ & $10(20)$ & $21(42)$ & 0.136 \\
\hline EM specialist & $13(9.3)$ & $32(22.9)$ & $35(25)$ & $46(32.9)$ & $14(10)$ & \\
\hline
\end{tabular}

(LRTI) (22.7\%). Those conditions did not differ across professional seniority (GPs, EM residents, and EM specialist) ( $p=0.1)$; however, the second common indication for physician group with less than five years of practice was urinary tract infections (UTI). The most commonly prescribed antibiotics were penicillin and cephalosporins (56.7\% and $31.9 \%$, respectively); this did not differ between the specialty level $(p=0.2)$, but there was a significant difference between the antibiotic choice and clinical practice $(p=0.02)$. The preferred antibiotic choices of physicians who had less than 15 years of practice were similar to those of all participants, but there was no difference between the groups of antibiotics chosen by the physicians who had more than 15 years of practice (Table 1).
Most of the participants noted that they were aware of the rapidly spreading antibiotic resistance worldwide (excellent 59.2\%, good $30.1 \%)$. More than half of them think that antibiotics were often prescribed inappropriately (62.1\%), and some even mentioned that they sometimes (39\%) prescribed antibiotics inappropriately. On the other hand, $57.4 \%$ stated that they often prescribed antibiotics in accordance with the guidelines for the correct indications, and this did not change with the professional seniorities $(p=0.3)$ or the level of training $(p=0.3$ ) (Tables 2 and 3). In any case, antibiotic stewardship awareness was poor among half of the participants (50.5\%), and there was a significant difference across the professional seniority $(p=0.03)$. It was better among the EM specialists group, $43.9 \%$ of whom noted 
Table 3. Responses of the participants to the questions related to their beliefs and attitudes toward antibiotic prescription and resistance Questions

Responses of participants to the questions about the antibiotic resistance and inappropriate prescription

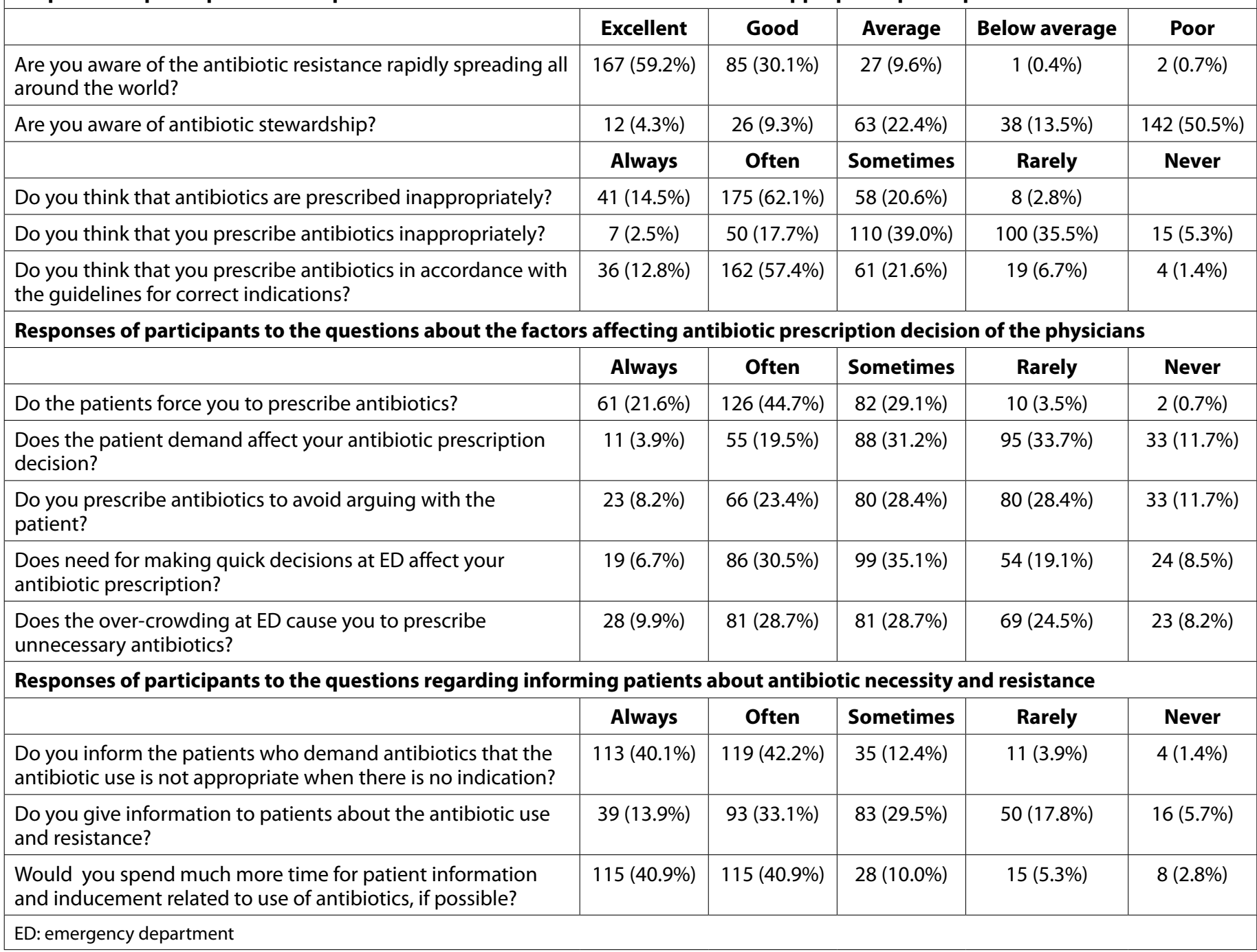

their awareness level of stewardship was average as well as above average (Tables 2 and 3 ).

There were also questions related to the factors affecting the physician's opinion during prescription. We asked whether the patients force the physician to prescribe antibiotics; 126 (44.7\%) of participants answered "often," and 61 (21.6\%) of participants answered "always." However, it was seen that patient demand did not significantly affect the physician's decision. Nearly one-third of the participants answered the question related to this "rarely" (33.7\%) and one-third "sometimes" (31.2\%) (Table 3). This condition did not differ between the professional seniority $(p=0.4)$ and the gender $(p=0.1)$, but it changed with clinical practice $(p=0.03)$, while physicians who were newer to the profession ( $<5$ years) noted that patient demand affected their decision more often (31.5\%) (Tables 2 and 4). We also asked if physicians prescribed antibiotics to avoid arguing with the patients. Attitudes did not differ across gender $(p=0.1)$, but differed across professional seniority $(p=0.01)$ and levels of training $(p=0.003)$. This behavior was more common among the
EM residents and physicians with less clinical practice (Tables 3 and 4). When questioned about the other factors, which were whether they had to make quick decisions at the ED or overcrowding of the ED caused inappropriate antibiotic prescription, there was no relation between the responses and professional seniority ( $p=0.7$ and $p=0.1$, respectively) (Table 2 ). Also associated with the clinical experience, there was no significant difference between the responses to the question, if need for making quick decisions affects physicians antibiotic prescription; however in connection with the overcrowding of the ED's effect on antibiotic prescription, there was an inverse relationship with the clinical experience $(p=0.07$ and $\mathrm{p}=0.01$, respectively) (Table 4 ).

Other questions were related to informing patients about the necessity for antibiotics and resistance. More than $80 \%$ of the participants noted that they informed patients who demanded antibiotics that it was inappropriate when there was no indication. Related to the antibiotic resistance, percentages of physicians giving information about this topic were lower. However, almost all of 
Table 4. Relations between the responses and clinical practice of the physicians

Questions

\begin{tabular}{l|l} 
Clinical practice & $n^{*}(\%)$
\end{tabular}

Do you think that you prescribe antibiotics in accordance with the guidelines for correct indications?

\begin{tabular}{|l|c|c|c|c|c|}
\hline & Never & Rarely & Sometimes & Often & Always \\
\hline 0-5 years & $5(6.8)$ & $18(24.7)$ & $43(58.9)$ & $7(9.6)$ \\
\hline $5-10$ years & $11(9.7)$ & $29(25.7)$ & $60(53.1)$ & $13(11.5)$ \\
\hline $10-15$ years & $6(10.2)$ & $8(13.6)$ & $33(55.9)$ & $12(20.3)$ \\
\hline $15+$ years & $1(2.7)$ & $6(16.2)$ & $26(70.3)$ & $4(10.8)$ & 0.3 \\
\hline
\end{tabular}

Does the patient demand affect your antibiotic prescription decision?

\begin{tabular}{|l|c|c|c|c|c|}
\hline & Never & Rarely & Sometimes & Often & Always \\
\hline 0-5 years & $7(9.6)$ & $17(23.3)$ & $26(35.6)$ & $23(31.5)$ \\
\hline $5-10$ years & $11(9.7)$ & $35(31)$ & $38(33.6)$ & $29(25.7)$ \\
\hline $10-15$ years & $11(18.6)$ & $23(39)$ & $16(27.1)$ & $9(15.3)$ \\
\hline $15+$ years & $4(10.8)$ & $20(54.1)$ & $8(21.6)$ & $5(13.5)$ & \\
\hline
\end{tabular}

Do you prescribe antibiotics to avoid arguing with the patient?

\begin{tabular}{|l|c|c|c|c|c|c|}
\hline & Never & Rarely & Sometimes & Often & Always & $11(15.1)$ \\
\hline 0-5 years & $5(6.8)$ & $14(19.2)$ & $23(31.5)$ & $20(27.4)$ & $32(28.3)$ & $8(7.1)$ \\
\hline 5-10 years & $12(10.6)$ & $25(22.1)$ & $36(31.9)$ & $13(22)$ & $11(18.6)$ & $3(5.1)$ \\
\hline $10-15$ years & $9(15.3)$ & $23(39)$ & $8(21.6)$ & $3(8.1)$ & $1(2.7)$ & \\
\hline $15+$ years & $7(18.9)$ & $18(48.6)$ & 8003 \\
\hline
\end{tabular}

Does need for making quick decisions at the emergency department affect your antibiotic prescription?

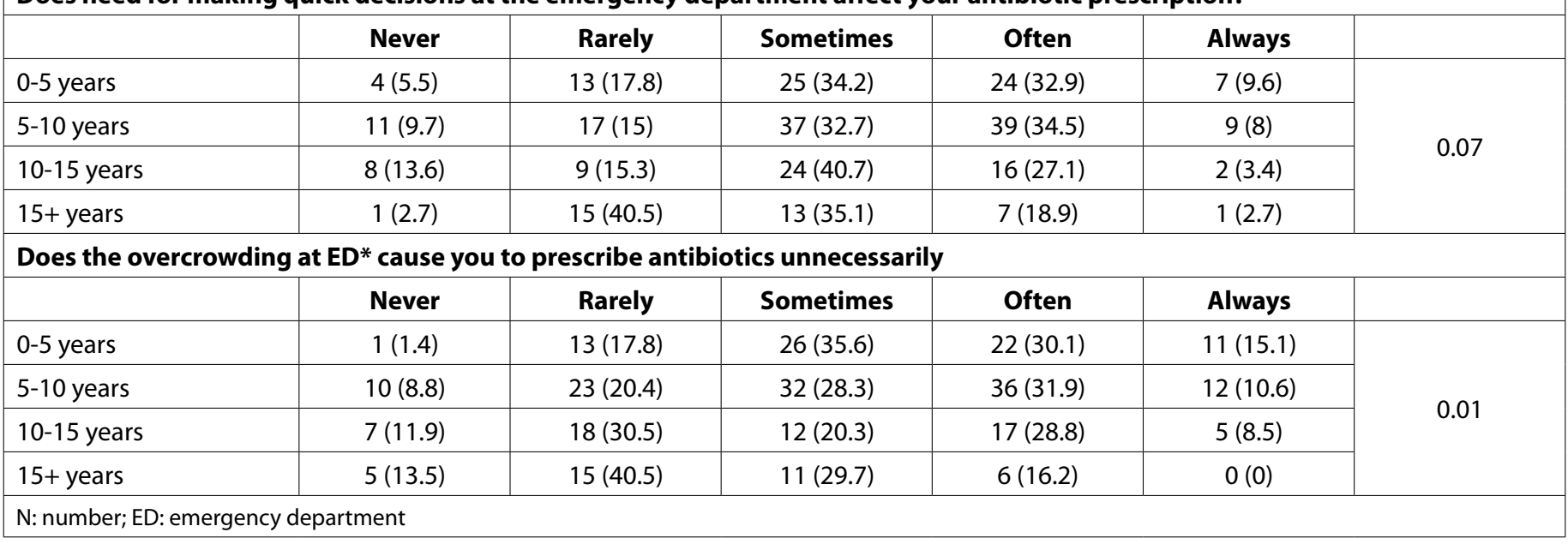

the participants assumed that they would spend much more time informing and inducing patients if possible (Table 3).

\section{Discussion}

This study demonstrated that $95 \%$ of the physicians agreed that they occasionally prescribed antibiotics unnecessarily. Almost all the physicians stated that patients forced them to prescribe antibiotics; however, this situation did not affect the prescription decision of the physician as much as predicted. Other important factors that affected physicians' antibiotic prescription decision were avoiding discussion with the patient and overcrowding of the ED, and their effects were proportionally reduced with the duration of the clinical practice of the physician.
Our survey confirmed that URTI was the most common indication for antibiotic prescription, and the most commonly prescribed antibiotics were penicillin and cephalosporins. Our results were similar to those of other studies in the literature. A study from China demonstrated that $90 \%$ of URTI prescriptions include antibiotics, and even $21 \%$ of them include a combination of antibiotics (7). Kho et al. (8) also demonstrated that $64.8 \%$ of patients with URTI received antibiotic prescription, but the chosen antibiotics changed across the countries. In a cohort study from Denmark, the most commonly prescribed antibiotic was penicillin $\mathrm{V}$, which accounted for $58 \%$ of all prescriptions, followed by macrolides (18\%) (9). However, in another study from Qatar, cephalosporin group was in the first line of treatment (43\%), followed by penicillins (28\%) (10). 
Antibiotic resistance is a growing public health problem, and almost all participants noted that they were aware of this. It is the main cause of morbidity and mortality from otherwise treatable infections, and it is largely attributed to the inappropriate use of antimicrobials (11). Nearly $40 \%$ of the participants noted that they sometimes prescribe antibiotics inappropriately. A study by Timbrook et al. (12) demonstrated that $39 \%$ of prescribed antibiotics at the ED did not meet the criteria for appropriateness; also, among appropriate indications, $13.8 \%$ had inappropriate dosing, duration, or expense. Antibiotic stewardship programs (ASP) are a set of interventions aiming at prescribing antibiotics appropriately and responsibly (6). Unfortunately, half of the participants were not aware of these. Dinh et al. (13) demonstrated in their study that ASP markedly decreased the number of unnecessary antimicrobial prescriptions, but it had little impact on most other aspects of the inappropriate prescription at the ED.

More than half of the participants assumed that patients force them to prescribe antibiotics. Our survey demonstrated that this insistent demand could sometimes affect the physician's decision, especially among those who were newer to the profession. In a study from Egypt, it was demonstrated that among patients with acute respiratory tract infections, $83 \%$ had been prescribed at least one antibiotic, and patient demand was a factor leading to over-prescription (14). Pan et al. (2) organized a virtual reality study, and it showed that eight out of nine trainees prescribed antibiotics, whereas seven out of the 12 GPs did so. The same study demonstrated that GPs were more likely to withstand the pressure to prescribe antibiotics than trainee doctors (2). According to a study by Gidengil et al. (15), 59\% of the participants noted that patient demand was a problem in their practice.

Another problem affecting the physician's decisions during drug prescription was overcrowding of the ED, which forced the physician to make quick decisions. Almost one-third of the participants noted that those factors "often" affect their decision, and one-third noted the frequency as "sometimes." Gidengil et al. (15) had similar results. They demonstrated that about one-third of clinicians reported feeling rushed almost always or most of the time.

The reason for patients' antibiotic demand can be attributed to their inadequate knowledge related to this topic. According to the results of a systematic review, 53.9\% of the sample did not know that antibiotics are not effective against viruses. Furthermore, although $59.4 \%$ of the sample was aware of antibiotic resistance, $26.9 \%$ of them did not know that misuse of antibiotics can lead to this problem (16). As Davis et al. (17) also demonstrated, patients reported experiencing confusion about which illnesses may be treated by antibiotics and unclear communication from clinicians about the appropriate use of antibiotics. In our study, $80 \%$ of the participants noted that they gave information to their patients related to the appropriate use of antibiotics, and almost all of them assumed that they would spend much more time for patient information and inducement if possible.

\section{Study limitations}

The number of the respondents was very small compared with the total number of the physicians working at the EDs all around the country. Also, physicians participated on a voluntary basis; hence, the survey might represent only the group of physicians who were more interested in antibiotic use.

\section{Conclusion}

Our study demonstrated that all physicians thought antibiotics had been prescribed inappropriately, with even $95 \%$ of them accepting that they also inappropriately prescribe antibiotics from time to time. Patient demand, having to make quick decisions, and overcrowding of EDs were some of the factors that affect the antibiotic prescription decisions of physicians, and the effect of these factors was inversely proportional to increased clinical practice.

Ethics Committee Approval: Ethics committee approval was received for this study from the Ethics Committee of Batman Region Community Hospital (Date 08/11/2017/File no: 75144452-000-3570).

Informed Consent: Written informed consent was not obtained from patients, because this survey was applied to E.M. physicians not to the patient and we also sent information related to aim of the study to participants as well.

Peer-review: Externally peer-reviewed.

Author Contributions: Concept - G.C.I.; Design - G.C.I.; Supervision - E.E.; Resources - G.C.I.; Materials - E.E.; Data Collection and/or Processing - G.C.I.; Analysis and/or Interpretation - G.C.I.; Literature Search - G.C.I.; Writing Manuscript - G.C.I.; Critical Review - G.C.I., E.E.

Conflict of Interest: The authors have no conflict of interest to declare.

Financial Disclosure: The authors declared that this study has received no financial support.

\section{References}

1. World Health Organization. Antimicrobial resistance: global report on surveillance. World Health Organization, 2014.

2. Pan $X$, Slater $M$, Beacco $A$, Navarro $X$, Rivas $A B$, Swapp $D$, et al. The Responses of medical general practitioners to unreasonable patient demand for antibiotics-A study of medical ethics using immersive virtual reality. PLoS One. 2016; 11: e0146837. [CrossRef]

3. Available from: https://www.statista.com/chart/4920/the-worldsbiggest-consumers-of-antibiotics/;2016 (accessed 2017 November 20)

4. Alothman A, Algwizani A, Alsulaiman M, Alalwan A, Binsalih S, Bosaeed $M$. Knowledge and attitude of physicians toward prescribing antibiotics and the risk of resistance in two reference hospitals. Infect Dis (Auckl). 2016; 9: 33-8. [CrossRef]

5. Bagger K, Nielsen AS, Siersma V, Bjerrum L. Inappropriate antibiotic prescribing and demand for antibiotics in patients with upper respiratory tract infections is hardly different in female versus male patients as seen in primary care. Eur J Gen Pract. 2015; 21: 118-23. [CrossRef]

6. Pulcini C. Antibiotic stewardship: a European perspective. FEMS Microbiol Lett. 2017; 364: DOI: 10.1093/femsle/fnx230. [CrossRef]

7. Yang L, Liu C, Wang L, Yin X, Zhang X. Public reporting improves antibiotic prescribing for upper respiratory tract infections in primary care: a matched-pair cluster-randomized trial in China. Health Res Policy Syst. 2014; 12: 61. [CrossRef]

8. Kho BP, Ong CM, Tan FT, Wee CY. Antibiotic prescribing for upper respiratory tract infections in sarawak district hospitals. Med J Malaysia. 2013; 68: 136-40.

9. Aabenhus R, Hansen MP, Saust LT, Bjerrum L. Characterisation of antibiotic prescriptions for acute respiratory tract infections in Danish general practice: a retrospective registry based cohort study. NPJ Prim Care Respir Med. 2017; 27: 37. [CrossRef]

10. Butt AA, Navasero CS, Thomas B, Marri SA, Katheeri HA, Thani AA, et al. Antibiotic prescription patterns for upper respiratory tract infections in 
the outpatient Qatari population in the private sector. Int J Infect Dis. 2017; 55: 20-3. [CrossRef]

11. Erku DA, Mekuria AB, Belachew SA. Inappropriate use of antibiotics among communities of Gondar town, Ethiopia: a threat to the development of antimicrobial resistance. Antimicrob Resist Infect Control. 2017; 6: 112. [CrossRef]

12. Timbrook TT, Caffrey AR, Ovalle A, Beganovic M, Curioso W, Gaitanis M, et al. Assessments of opportunities to Improve antibiotic prescribing in an emergency department: A period prevalence survey. Infect Dis Ther. 2017; 6: 497-505. [CrossRef]

13. Dinh A, Duran C, Davido B, Bouchand F, Deconinck L, Matt M, et al. Impact of an antimicrobial stewardship programme to optimize antimicrobial use for outpatients at an emergency department. J Hosp Infect. 2017; 97: 288-93. [CrossRef]
14. Kandeel A, El-Shoubary W, Hicks LA, Fattah MA, Dooling KL, Lohiniva $A L$, et al. Patient attitudes and beliefs and provider practices regarding antibiotic use for acute respiratory tract infections in Minya, Egypt. Antibiotics (Basel.) 2014; 3: 632-44. [CrossRef]

15. Gidengil CA, Mehrotra A, Beach S, Setodji C, Hunter G, Linder JA. What drives variation in antibiotic prescribing for acute respiratory infections? J Gen Intern Med. 2016; 31: 918-24. [CrossRef]

16. Gualano MR, Gili R, Scaioli G, Bert F, Siliquini R. General population's knowledge and attitudes about antibiotics: a systematic review and meta-analysis. Pharmacoepidemiol Drug Saf. 2015; 24: 2-10. [CrossRef]

17. Davis ME, Liu TL, Taylor YJ, Davidson L, Schmid 5, Yates T, et al. Exploring patient awareness and perceptions of the appropriate use of antibiotics: A mixed-methods study. Antibiotics (Basel). 2017; 6: pii: E23. [CrossRef] 
Appendix 1. Does patient demand affect the physicians' antibiotic prescription decision in emergency departments?

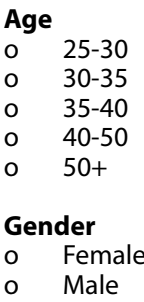

What is your professional status?

o General practitioner

o Emergency medicine resident

o Emergency medicine specialist

How long is your clinical practice?

$$
0-5
$$

5-10

10-15

$15-20$

$20+$

How many patients do you see per day?

$$
\begin{aligned}
& 0-20 \\
& 20-50 \\
& 50-100 \\
& 100-200
\end{aligned}
$$

$200+$

For which indication do you prescribe antibiotics most?

o Upper respiratory tract infection

Lower respiratory tract infection

Urinary tract infection

Acute gastrointestinal infection

Others

Which group of antibiotics do you prescribe most?

Penicillins

$$
\begin{aligned}
& \text { Cephalosporins } \\
& \text { Macrolides } \\
& \text { Quinolones }
\end{aligned}
$$

Others

Do you think that antibiotics are prescribed inappropriately?

o Always

Often

Sometimes

Rarely

Never

Do you aware of the antibiotic resistance rapidly-spreading all around the world?

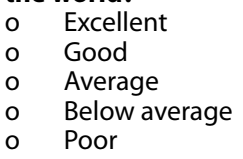

Do the patients force you to prescribe antibiotics?

$\begin{array}{ll}\text { o } & \text { Always } \\ \text { o } & \text { Often } \\ \text { o } & \text { Sometimes } \\ \text { o } & \text { Rarely } \\ \text { o } & \text { Never }\end{array}$

Do you think that you prescribe antibiotics inappropriately?

Always

Often

Sometimes

Rarely

Never

Does the patient demand affect your antibiotic prescription decision?

- Always

Often

Sometimes

Rarely

Never
Do you aware of antibiotic stewardship?

- Excellent

Good

Average

Below average

Poor

Does having to make quick decisions at emergency department affect your antibiotic prescription?

o Always

Often

Sometimes

Rarely

o Never

Do you give information to patients with antibiotic demands that it is not appropriate when there is no indication?

o Always

Often

Sometimes

Rarely

Never

Do you give information to patients related to antibiotic use and resistance?

- Always

Often

Sometimes

Rarely

Never

Does the patient's persistent attitude affect your decision?

$$
\begin{array}{ll}
\text { o } & \text { Always } \\
\text { o } & \text { Often } \\
\text { o } & \text { Sometimes } \\
\text { o } & \text { Rarely } \\
\text { o } & \text { Never }
\end{array}
$$

How often antibiotics are prescribed inappropriately according to you? o Very often

o Often

- Average

o Rare

o Very rare

Do you spend much more time for patient information and inducement if possible?

o Always

Often

Sometimes

Rarely

Never

Does the overcrowding at ED cause you to prescribe antibiotics unnecessarily

- Always

o Often

- Sometimes

- Rarely

o Never

Would you prescribe antibiotics to avoid arguing with the patient?

o Always

Often

Sometimes

Rarely

o Never

Do you think that you prescribe antibiotics in accordance with the guidelines for the correct indications?

o Always

Often

Sometimes

Rarely

o Never 